Pacific Journal of Mathematics

ON ABSOLUTELY CONTINUOUS FUNCTIONS AND THE 


\title{
ON ABSOLUTELY CONTINUOUS FUNCTIONS AND THE WELL-BOUNDED OPERATOR
}

\author{
W. H. SILLS
}

The author considers an operator $T$ in a reflexive Banach space $X$ for which there is a bounded operational calculus $a \rightarrow a(T)$ defined on $A C(I)$, the algebra of absolutely continuous functions defined on $I=[0,1]$ with the norm $|a(0)|+\operatorname{Var}_{I}(a)$ for $a \in A C(I)$. Such operators, called well-bounded, have been investigated by Smart and Ringrose (J. Australian Math. Soc. 1 (1960), 319-343 and Proc. London Math. Soc. (3) 13 (1963), 613-638). The present paper explores a new method for obtaining the spectral theorem for this operator. Let $A C_{0}$ be the maximal ideal of members of $A C(I)$ which are zero at 0 . The method consists in introducing Arens multiplication into $A C_{0}^{* *}$, the second conjugate space of $A C_{0}$, and in investigating the larger algebra for a suitable family of idempotents which will serve as candidates for bounded spectral projections associated with $T$. Idempotents in $A C_{0}^{* *}$ are mapped into these projections by means of a homomorphism extension technique which extends the original operational calculus of $A C_{0}$ into $B(X)$ (the bounded linear operators on $X$ ), to a bounded homomorphism of $A C_{0}^{* *}$ into $B(X)$. The extended homomorphism is defined on a quotient algebra of $A C_{0}^{* *}$. This quotient algebra turns out to be a copy of all functions of bounded variation on $I$ which are zero at 0 under the usual pointwise operations.

Let $A C(I)$ be the complex algebra of complex-valued, absolutely continuous functions on $I=[0,1]$ with the algebraic operations being the usual addition and multiplication of functions. This algebra is a Banach algebra under the norm (see Section 3)

$$
\|a\|=|a(0)|+\operatorname{Var}_{I}(a),
$$$$
a \in A C(I) \text {. }
$$

We shall consider a linear operator $T$ in a reflexive Banach space $X$ for which there is an operational calculus $a \rightarrow a(T)$ satisfying

$$
\|a(T)\| \leqq K\|a\|, \quad \cdot a \in A C(I) .
$$

This operator, an example of a well-bounded operator, was introduced by Smart [14]. Smart showed that $T$ determines a bounded, strongly

Received July 9, 1964, and in revised form January 9, 1965. This work was partially supported by the Air Force Office of Scientific Research, under Contract AF 49 (638) 859 and Grant AF-AFOSR 62-140, This paper is a portion of the author's doctoral dissertation at the University of California at Berkeley (1963) which was written under the supervision of Professor W. G. Bade. 
continuous family of projections $\left\{E_{t}\right\}$, indexed on the real line, and he proved the existence of the scalar operator

$$
S x=\int t d E_{t} x,
$$

$x \in X$.

Ringrose [12] established that $S=T$ and has recently [13] given a more comprehensive treatment in which he considers the nonreflexive situation.

In the present paper we explore a new method for obtaining the spectral theorem for this operator in a reflexive Banach space. Let $A C_{0}$ be the maximal ideal of members of $A C(I)$ which are zero at 0 . Our method consists in introducing Arens multiplication into $A C_{0}^{* *}$, the second conjugate space of $A C_{0}$, and in investigating the larger algebra for a suitable family of idempotents which will serve as candidates for Smart's projections. The algebra $A C_{0}^{* *}$ is neither commutative nor semi-simple. Idempotents in this algebra are mapped into projections in $B(X)$, the algebra of bounded operators on $X$, by means of a homomorphism extension technique which extends the original operational calculus $a \rightarrow a(T)$ defined on $A C_{0}$ to a bounded homomorphism of $A C_{0}^{* *}$ into $B(X)$. Kamowitz has used this extension procedure in [9]. When $X$ is reflexive, the extended homomorphism is defined on a quotient algebra of $A C_{0}^{* *}$. This quotient algebra is a copy of $B V_{0}$, the algebra of functions of bounded variation on $I$ which are zero at 0 (cf. [15]).

The algebra $A C_{0}$ and its conjugate spaces are discussed in Sections 3 through 5. The extension theorem is in Section 6 and Section 7 is concerned with the well-bounded operator.

2. Preliminary notions. If $X$ is a Banach space, $X^{*}$ and $X^{* *}$ will denote the conjugate space and second conjugate space of $X$, respectively. The natural embedding of $X$ into $X^{* *}$ will be written $x \rightarrow \widehat{x}$, where $\widehat{x}\left(x^{*}\right)=x^{*}(x)$ for $x^{*}$ in $X^{*}$. It is well known that $\hat{X}$ is dense in $X^{* *}$ when the latter space is provided with the weak*topology [4, p. 425]. Let $A$ be a Banach algebra, with unit or not, commutative or not, with elements $a, b, \cdots$. Let the elements of $A^{*}$ be written $f, g, \cdots$. Denote those of $A^{* *}$ by $\xi, \eta, \cdots$. Arens multiplication is introduced into $A^{* *}$ in three stages as follows: for $f \in A^{*}$ and $a \in A, f \odot a \in A^{*}$ is defined by $(f \odot a)(b)=f(a b)$, for $b \in A$. If $\eta \in A^{* *}$ and $f \in A^{*}, \eta \odot f \in A^{*}$ is defined by $(\eta \odot f)(a)=\eta(f \odot a)$, for $a \in A$. Finally, let $\xi, \eta \in A^{* *}$ be given. Then $\xi \odot \eta \in A^{* *}$ is defined by $(\xi \odot \eta)(f)=\xi(\eta \odot f)$, for $f \in A^{*}$. It follows that $\|\xi \odot \eta\| \leqq\|\xi\|\|\eta\|$. The map $\odot$ is bounded and bilinear at each stage of definition. As is noted in $[1,2], \odot$ is an associative multiplication in $A^{* *}$, making this space into a Banach algebra under the usual norm. The natural 
map $a \rightarrow \widehat{a}$ is an isometric algebraic isomorphism, and if $A$ is commutative, $A$ is in the center of $A^{* *}$. Finally, we recall that for fixed $\eta \in A^{* *}$, the product $\xi \odot \eta$ is weak ${ }^{*}$-continuous in $\xi \in A^{* *}$, and for fixed $a \in A, \hat{a} \odot \eta$ is weak*-continuous in $\eta \in A^{* *}$. We note that $A^{* *}$ need not be commutative nor semi-simple even when $A$ is (see Sec. 4, [3]).

Let $X, Y$ and $Z$ be Banach spaces. Let $\Delta$ be a bilinear map of $X \times Y$ into $Z$ (written $(x, y) \rightarrow x \Delta y$ ) and suppose that

$$
\|\Delta\|=\sup \{\|x \Delta y\|:\|x\|=\|y\|=1\}
$$

is finite. Following Arens [2], we define the adjoint of $\Delta$ by the $\operatorname{map} \Delta^{*}: Z^{*} \times X \rightarrow Y^{*}\left(\left(z^{*}, x\right) \rightarrow z^{*} \Delta^{*} x\right)$ where we put $\left(z^{*} \Delta^{*} x\right)(y)=$ $z^{*}(x \Delta y)$, for $z^{*} \in Z^{*}, x \in X$, and $y \in Y$. It is easy to see that $\Delta^{*}$ is bilinear and has the same norm as $\Delta$. In later sections we shall always consider a second conjugate space of a Banach algebra as a Banach algebra.

3. Absolutely continuous functions. Let $A C_{0}$ be the complex algebra of absolutely continuous complex-valued functions on $I=[0,1]$ which are zero at 0 , with the usual multiplication and addition for functions. In this section, the formula for the Arens multiplication in $A C_{0}^{* *}$ is derived in terms of corresponding finitely additive set functions.

Let $L_{1}$ be the complex space $L_{1}\{I, \mathscr{L}, m\}$ where $\mathscr{L}$ is the $\sigma$-algebra of Lebesgue measurable subsets of $I$ and $m$ is Lebesgue measure. Consider the norm on $A C_{0}$ given by

$$
\|a\|=\int_{I}\left|a^{\prime}(s)\right| d s=\operatorname{Var}_{I}(a), \quad a \in A C_{0},
$$

where $a^{\prime}$ is the almost everywhere derivative of $a$. The map $a \rightarrow a^{\prime}$ is an isometric isomorphism between $A C_{0}$ with this norm and $L_{1}$. A crude estimate yields $\|a b\| \leqq 2\|a\|\|b\|$. This norm is actually submultiplicative on $A C_{0}$.

Lemma 3.1. Under the above norm, $A C_{0}$ is a Banach algebra.

Proof. It is convenient to prove the lemma in $L_{1}$. Let

$$
(f \circ g)(t)=f(t) \int_{0}^{t} g(s) d s+g(t) \int_{0}^{t} f(s) d s,
$$

for $f, g$ in $L_{1}$ and $t \in I$. This is a copy of the multiplication in $A C_{0}$. We must show that $\|f \circ g\|_{1} \leqq\|f\|_{1}\|g\|_{1}$, where $\|\cdot\|_{1}$ denotes the $L_{1}$ norm. The product $f \circ g$ is continuous in $f$ and $g$ since $\|f \circ g\|_{1} \leqq$ 
$2\|f\|_{1}\|g\|_{1}$. Hence, it is sufficient to verify the inequality we want for linear combinations of characteristic functions of intervals as these are dense in $L_{1}$. Suppose that whenever $e$ and $d$ are intervals from $I$, $\left\|k_{e} \circ k_{d}\right\|_{1} \leqq\left\|k_{e}\right\|_{1}\left\|k_{d}\right\|_{1}$, where $k_{e}$ and $k_{d}$ are the characteristic functions of $e$ and $d$. If $f=\sum \alpha_{i} k_{e_{i}}$ and $g=\sum \beta_{j} k_{d_{j}}$, where the intervals $e_{i}$ are pairwise disjoint and the intervals $d_{j}$ are pairwise disjoint, then $\|f\|_{1}=\sum\left|\alpha_{i}\right|\left\|k_{e_{i}}\right\|_{1}$ and a similar statement holds for $g$. Thus,

$$
f \circ g=\left(\sum \alpha_{i} k_{e_{i}}\right) \circ\left(\sum \beta_{j} k_{a_{j}}\right)=\sum \sum \alpha_{i} \beta_{j}\left(k_{e_{i}} \circ k_{a_{j}}\right)
$$

and

$$
\|f \circ g\|_{1} \leqq \sum \sum\left|\alpha_{i}\left\|\beta_{j} \mid\right\| k_{e_{i}} \circ k_{\alpha_{j}}\left\|_{1} \leqq\right\| f\left\|_{1}\right\| g \|_{1}\right. \text {. }
$$

Hence, it is enough to establish the inequality for two characteristic functions of intervals. This is straightforward and will be omitted.

If the unit function $e(t)=1$ is adjoined to $A C_{0}$, we obtain all absolutely continuous functions on $I, A C(I)=A C_{0} \oplus\{\lambda e\}, \lambda$ complex. This algebra is a Banach algebra under the norm $\|b\|=|b(0)|+\operatorname{Var}(b)$ by Lemma 3.1. Each maximal ideal of $A C(I)$ consists of all of those functions which vanish at a point $t \in I([10$, the lemma on page 55]). Equivalently, each multiplicative linear functional on $A C(I)$ is a point evaluation $b \rightarrow b(t)$.

LEMMA 3.2. The nonzero multiplicative linear functionals on $A C_{0}$ are of the form $\mu_{t}(\alpha)=a(t), 0<t \leqq 1, a \in A C_{0}$.

Proof. If $\mu$ is a multiplicative linear functional on $A C_{0}$, it extends to a multiplicative linear functional $\sigma$ on $A C_{0} \oplus\{\lambda e\}$ given by $\sigma(a+\lambda e)=$ $\mu(\alpha)+\lambda$. Since $\sigma$ is a point evaluation, $\mu$ is a point evaluation as stated.

The spaces $A C_{0}, A C_{0}^{*}$ and $A C_{0}^{* *}$ may be identified, respectively, with the spaces $L_{1}, L_{\infty}$ and $L_{\infty}^{*}$, where $L_{\infty}$ is the complex space $L_{\infty}\{I, \mathscr{L}, m\}$ with essential supremum norm which we will denote by $N_{\infty}(\cdot)$. It is well known that $L_{\infty}^{*}$ is isometrically isomorphic with the complex Banach space $b a\{I, \mathscr{L}, m\}$ consisting of all finitely additive, complexvalued set functions $\xi$ defined on $\mathscr{L}$, which vanish on Lebesgue null sets and which have finite total variation on $I$ with respect to $\mathscr{L}$ ([4, p. 296]). The total variation of $\xi$ on a set $E \in \mathscr{L}$, with respect to $\mathscr{L}$, is given by

$$
\operatorname{Var}_{\mathscr{L}}(\xi, E)=\sup \Sigma\left|\xi\left(E_{i}\right)\right|
$$

where the supremum is taken over all partitions of $E$ into a finite union of mutually disjoint sets $E_{i}$ from $\mathscr{L}$. 
In order to simplify notation we will use the same symbol for corresponding elements in equivalent spaces. Whether a symbol denotes a functional or a point or set function should be evident from the context. Thus, we have the following formulas:

$$
\begin{aligned}
& f(a)=\int_{I} f(s) a^{\prime}(s) d s, \quad f \in A C_{0}^{*}, a \in A C_{0} \\
& \xi(f)=\int_{I} f(s) d \xi(s), \quad f \in A C_{0}^{*}, \xi \in A C_{0}^{* *}
\end{aligned}
$$

where $\|f\|=N_{\infty}(f)$ and $\|\xi\|=\operatorname{Var}_{\mathscr{L}}(\xi, I)$.

The notion of integration of $L_{\infty}$ functions with respect to finitely additive set functions as in formula (3.2.1) may be defined as follows: for $\xi \in b a\{I, \mathscr{L}, m\}, f \in L_{\infty}, E \in \mathscr{L}$, let

$$
\int_{E} f(s) d \xi(s)=\lim _{n} \int_{E} f_{n}(s) d \xi(s)
$$

where $f_{n}(s)=\sum \alpha_{i n} k_{E_{i n}}(s), n=1,2,3, \cdots$, is a sequence of finite linear combinations of characteristic functions of disjoint sets from $\mathscr{L}$ such that $N_{\infty}\left(f-f_{n}\right) \rightarrow 0$ and where

$$
\int_{E} f_{n}(s) d \xi(s)=\sum \alpha_{i n} \xi\left(E \cap E_{i n}\right)
$$

This integral is finitely additive on $\mathscr{L}$ and

$$
\left|\int_{E} f(s) d \xi(s)\right| \leqq N_{\infty}(f) \operatorname{Var}_{\mathscr{L}}(\xi, E) .
$$

The formulas for the Arens multiplication are computed next.

Lemma 3.3. If $f \in A C_{0}^{*}, a \in A C_{0}$, then for almost all $t \in I$,

$$
(f \odot a)(t)=\int_{t}^{1} f(s) a^{\prime}(s) d s+f(t) a(t) .
$$

Proof. Let $F(t)=\int_{t}^{1} f(s) a^{\prime}(s) d s$. Then $F^{\prime}(t)=-f(t) a^{\prime}(t)$ for almost all $t \in I$. If $b \in A C_{0}$ is arbitrary,

$$
\begin{aligned}
(f \odot a)(b) & =f(a b)=\int_{I} f(t) a^{\prime}(t) b(t) d t+\int_{I} f(t) a(t) b^{\prime}(t) d t \\
& =-\int_{I} F^{\prime}(t) b(t) d t+\int_{I} f(t) a(t) b^{\prime}(t) d t
\end{aligned}
$$

Since $F$ and $b$ are absolutely continuous, we may integrate the integral containing $F^{\prime}$ by parts to obtain 


$$
\begin{gathered}
-F(t) b(t)]_{0}^{1}+\int_{I} F(t) b^{\prime}(t) d t+\int_{I} f(t) a(t) b^{\prime}(t) d t \\
=\int_{I}\{F(t)+f(t) a(t)\} b^{\prime}(t) d t .
\end{gathered}
$$

Thus, for arbitrary $b \in A C_{0}$,

$$
(f \odot a)(b)=\int_{I}\left\{\int_{t}^{1} f(s) a^{\prime}(s) d s+f(t) a(t)\right\} b^{\prime}(t) d t
$$

The lemma now follows.

LEMma 3.4. If $f$ is continuous on $I$ and $\xi \in b a\{I, \mathscr{L}, m\}$ and $h(s)=\xi((0, s))$ for $0<s \leqq 1, h(0)=0$, then

$$
\int_{I} f(s) d \xi(s)=\int_{I} f(s) d h(s)
$$

where the second integral is a Riemann-Stieltjes integral.

Proof. The total variation of $h$ on $I$,

$$
\operatorname{Var}_{I}(h)=\sup \left\{\sum\left|h\left(s_{i}\right)-h\left(s_{i-1}\right)\right|: 0=s_{0}<\cdots<s_{n}=1\right\}
$$

does not exceed $\operatorname{Var}_{\mathscr{L}}(\xi, I)$, by definition of $h$ and the finite additivity of $\xi$. By uniform continuity, $f$ can be approximated uniformly on $I$ by step functions of the form

$$
f_{\varepsilon}=\sum f\left(s_{i}^{\prime}\right) k_{e_{i}}, \quad N_{\infty}\left(f-f_{\varepsilon}\right)<\varepsilon
$$

where $e_{i}$ is an interval with end points $s_{i-1}, s_{i} ; s_{i}^{\prime} \in e_{i}$ and $0=$ $s_{0}<\cdots<s_{n}=1$. The integral of $f_{\varepsilon}$ with respect to $\xi$ is the RiemannStieltjes sum

$$
S=\sum f\left(s_{i}^{\prime}\right)\left[h\left(s_{i}\right)-h\left(s_{i-1}\right)\right]
$$

and

$$
\left|S-\int_{I} f(s) d \xi(s)\right|=\left|\int_{I}\left(f_{\varepsilon}-f\right)(s) d \xi(s)\right| \leqq \varepsilon\|\xi\|
$$

Lemma 3.5. If $\eta \in A C_{0}^{* *}$ and $f \in A C_{0}^{*}$, then for almost all $t \in I$,

$$
(\eta \odot f)(t)=\int_{t}^{1} f(s) d \eta(s)+\eta((0, t)) f(t) .
$$

Proof. Let $b \in A C_{0}$ be arbitrary. Then

$$
\begin{aligned}
(\eta \odot f)(b) & =\eta(f \odot b)=\int_{I}(f \odot b)(t) d \eta(t) \\
& =\int_{I}\left\{\int_{t}^{1} f(s) b^{\prime}(s) d s\right\} d \eta(t)+\int_{I} f(t) b(t) d \eta(t)=J_{1}+J_{2}
\end{aligned}
$$


by Lemma 3.3. Let $h(s)=\eta((0, s)), h(0)=0$. By Lemma 3.4, $J_{1}$ is the R.S. integral

$$
J_{1}=\int_{I}\left\{\int_{t}^{1} f(s) b^{\prime}(s) d s\right\} d h(t)
$$

After integrating by parts,

$$
J_{1}=\int_{I} \eta((0, t)) f(t) b^{\prime}(t) d t .
$$

For $J_{2}$, define

$$
x^{*}(g)=\int_{I} g(t) f(t) d \eta(t),
$$

Then $x^{*}$ is a bounded linear functional on $L_{\infty}$ and, therefore, there is a $\psi \in b a\{I, \mathscr{L}, m\}$ such that

$$
x^{*}(g)=\int_{I} g(t) d \psi(t),
$$

and $J_{2}=x^{*}(b)$. By Lemma 3.4 and making use of the absolute continuity of $b$, we may integrate $x^{*}(b)$ as expressed in (3.3.1) by parts to get

$$
\begin{aligned}
J_{2} & =b(t) \psi((0, t))]_{0}^{1}-\int_{I} \psi((0, t)) b^{\prime}(t) d t \\
& =b(1) \psi((0,1))-\int_{I} \psi((0, t)) b^{\prime}(t) d t=\int_{I} \psi((t, 1)) b^{\prime}(t) d t .
\end{aligned}
$$

Since

$$
x^{*}\left(k_{(t, 1)}\right)=\int_{t}^{1} f(s) d \eta(s)
$$

and $x^{*}\left(k_{(t, 1)}\right)=\psi((t, 1))$ from (3.3.1), then

$$
J_{2}=x^{*}(b)=\int_{I} \psi((t, 1)) b^{\prime}(t) d t=\int_{I}\left\{\int_{t}^{1} f(s) d \eta(s)\right\} b^{\prime}(t) d t .
$$

Therefore,

$$
(\eta \odot f)(b)=\int_{I}\left\{\eta((0, t)) f(t)+\int_{t}^{1} f(s) d \eta(s)\right\} b^{\prime}(t) d t
$$

for arbitrary $b \in A C_{0}$. This proves the lemma.

The multiplication in $A C_{0}^{* *}$ can now be put into concrete form.

THEOREM 3.6. If $\xi, \eta \in A C_{0}^{* *}$, the corresponding set function $\xi \odot \eta \in b a\{I, \mathscr{L}, m\}$ is given by 


$$
(\xi \odot \eta)(E)=\int_{I}\left\{k_{E}(t) \eta((0, t))+\eta(E \cap(t, 1))\right\} d \xi(t)
$$

for each $E \in \mathscr{L}$.

Proof. Write $(\xi \odot \eta)(E)=(\xi \odot \eta)\left(k_{E}\right)=\xi\left(\eta \odot k_{E}\right)$ and apply Lemma 3.5.

4. Idempotents. With the aid of Theorem 3.6 we can identify a large family of idempotents in $A C_{0}^{* *}$ and discuss their multiplication. In this section we will find a special family of idempotents useful for spectral theory.

Let $\Phi$ be the family of nonzero multiplicative linear functionals on $L_{\infty}$. Each $\varphi \in \Phi$, when viewed as a member of $b a\{I, \mathscr{L}, m\}$, is a set function on $\mathscr{L}$ which assumes only the values 0 and 1 . Let $\mathscr{R}$ denote the family of Lebesgue null sets contained in $\mathscr{L}$. There is a one-to-one correspondence between members of $\Phi$ and ultrafilters contained in $\mathscr{L} \sim \mathscr{R}$ given by

$$
U_{\varphi}=[E \in \mathscr{L}: \varphi(E)=1] \text {. }
$$

The next lemma is probably well known. A short proof is given for completeness.

LemMa 4.1. There is a continuous map $h$ from $\Phi$ with the weak*-topology onto $I$ such that for each $f$ continuous on $I, \varphi(f)=$ $f(h(\varphi)), \varphi \in \Phi$.

Proof. For each $\varphi \in \Phi$ define $h(\varphi)$ to be the point $t \in I$ to which the ultrafilter $U_{\varphi}$ converges. That is to say, $\{t\}=\bigcap[\bar{E}: E \in \mathscr{L}, \varphi(E)=1]$, where $\bar{E}$ is the closure of $E$ in $I$. This map is onto $I$, since, given $t \in I$, the filter base consisting of open neighborhoods of $t$ in $I$ is contained in $\mathscr{L} \sim \mathscr{R}$ and by Zorn's lemma is contained in an ultrafilter in $\mathscr{L} \sim \mathscr{R}$. If $\varphi$ is the set function corresponding to this ultrafilter, then $h(\varphi)=t$. To show $h$ is continuous, let $F$ be a closed neighborhood of $t$ in $I$ and let $\varphi_{t} \in \Phi$ where $h\left(\varphi_{t}\right)=t$. By the definition of $h$ it is obvious that the weak*-neighborhood of $\varphi_{t}$ given by $\left[\varphi \in \Phi:\left|\varphi(F)-\varphi_{t}(F)\right|<1\right]$ is mapped into $F$ under $h$. To conclude the proof, let $f$ be continuous on $I$ and let $h(\varphi)=t$. Then

$$
|\varphi(f)-f(t)| \leqq \int_{I}|f(s)-f(t)| d \varphi(s)=\int_{V}|f(s)-f(t)| d \varphi(s)
$$

where $V$ is an arbitrarily small neighborhood of $t$ in $I$.

DeFINITION 4.2. Let $F_{t}=[\varphi \in \Phi: h(\varphi)=t], t \in I$ denote the fiber at $t$. 
If $\varphi \in F_{t}$, we will write $\varphi=\varphi_{t}$. Each member of $F_{t}$ assumes the value 1 on each open neighborhood of $t$ in $I$. If $E \in \mathscr{L}$ and $t \notin \bar{E}$, then $\varphi_{t}(E)=0$ for each $\varphi_{t} \in F_{t}$.

In the next lemma we consider multiplication between members of fibers. We will adopt the convention that $\varphi_{0}((0,0))=\varphi_{1}((1,1))=0$.

LEMMA 4.3. If $0 \leqq s \leqq t \leqq 1, E \in \mathscr{L}$, and $\varphi_{s}, \varphi_{t}$ are members of $F_{s}, F_{t}$, respectively, then

$$
\begin{aligned}
& \left(\varphi_{s} \odot \varphi_{t}\right)(E)=\varphi_{s}(E) \varphi_{s}((t, 1))+\varphi_{t}(E) \varphi_{s}((0, t)) \\
& \left(\varphi_{t} \odot \varphi_{s}\right)(E)=\varphi_{t}(E) \varphi_{t}((s, 1))+\varphi_{s}(E) \varphi_{t}((0, s)) .
\end{aligned}
$$

Proof. First suppose that $0<s \leqq t<1$. By Theorem 3.6,

$$
\left(\varphi_{s} \odot \varphi_{t}\right)(E)=\int_{I}\left\{k_{E}(u) \varphi_{t}((0, u))+\varphi_{t}(E \cap(u, 1))\right\} d \varphi_{s}(u) .
$$

By the multiplicative property of $\varphi_{t}$, this is equal to

$$
\int_{E} \varphi_{t}((0, u)) d \varphi_{s}(u)+\varphi_{t}(E) \int_{I} \varphi_{t}((u, 1)) d \varphi_{s}(u) \text {. }
$$

Since $\varphi_{t}((0, u))=k_{(t, 1)}(u)$ and $\varphi_{t}((u, 1))=k_{(0, t)}(u)$ except at some endpoints, which can be ignored,

$$
\left(\varphi_{s} \odot \varphi_{t}\right)(E)=\int_{E \cap(t, 1)} d \varphi_{s}(u)+\varphi_{t}(E) \int_{(0, t)} d \varphi_{s}(u)
$$

and the first formula of (4.3.1) is apparent by the multiplicative property of $\varphi_{s}$. The other cases and the second formula are verified in a similar manner.

THEOREM 4.4. (i) Each $\varphi \in \Phi$ is an idempotent.

(ii) If $0 \leqq s<t \leqq 1$ and $\varphi_{s}, \varphi_{t}$ are members of $F_{s}, F_{t}$, then

$$
\varphi_{s} \odot \varphi_{t}=\varphi_{t} \odot \varphi_{s}=\varphi_{t} \text {. }
$$

Proof. (i) If $\varphi \in \Phi, \varphi=\varphi_{s}$ for some $s \in I$. By (4.3.1), $\left(\varphi_{s} \odot \varphi_{s}\right)(E)=\varphi_{s}(E)$ for each $E \in \mathscr{L}$.

(ii) Since $s<t, \varphi_{s}((t, 1))=0$ and $\varphi_{s}((0, t))=1$. Formula (4.3.1) implies that $\left(\varphi_{s} \odot \varphi_{t}\right)(E)=\varphi_{t}(E)$ for $E \in \mathscr{L}$. Similarly, as $\varphi_{t}((s, 1))=1$ and $\varphi_{t}((0, s))=0$, we have $\left(\varphi_{t} \odot \varphi_{s}\right)(E)=\varphi_{t}(E)$.

A finer classification of members of $\Phi$ will be needed. If $\varphi_{s} \in F_{s}$, it must assume the value 1 on exactly one of the intervals $(0, s)$ or $(s, 1)$, if $0<s<1$. We shall write $\varphi_{s}=\varphi_{s}^{+}$if $\varphi_{s}((s, 1))=1$ and $\varphi_{s}=\varphi_{s}^{-}$if $\varphi_{s}((0, s))=1$. At the end points of $I=[0,1]$, we must put $\varphi_{1}=\varphi_{1}^{-}$and $\varphi_{0}=\varphi_{0}^{+} ; \varphi_{1}^{+}$and $\varphi_{0}^{-}$are not defined. 
This classification splits each interior fiber into a positive and negative part, $F_{s}=F_{s}^{+} \cup F_{s}^{-}, 0<s<1$. The next theorem gives the Arens multiplication between elements of the same fiber. The noncommutativity of the multiplication is evident in (i) or (iii).

THEOREM 4.5. Let $\varphi_{t}^{+}, \psi_{t}^{+} \in F_{t}^{+}$and $\varphi_{t}^{-}, \psi_{t}^{-} \in F_{t}^{-}$for $t$ as indicated below. Then

$$
\begin{aligned}
& \text { (i) } \varphi_{t}^{+} \odot \psi_{t}^{+}=\varphi_{t}^{+} \text {if } 0 \leqq t<1, \\
& \text { (ii) } \varphi_{t}^{+} \odot \psi_{t}^{-}=\psi_{t}^{-} \odot \varphi_{t}^{+}=\varphi_{t}^{+} \text {if } 0<t<1 \text {, } \\
& \text { (iii) } \varphi_{t}^{-} \odot \psi_{t}^{-}=\psi_{t}^{-} \text {if } 0<t \leqq 1 .
\end{aligned}
$$

Proof. The proof is an immediate consequence of (4.3.1).

The multiplication between members of $\Phi$ may be summarized as follows: if $t<s$ in $I$, let $F_{t}$ preceed $F_{s}$. In a given fiber $F_{u}$, let $F_{u}^{-}$preceed $F_{u}^{+}$when defined. If $\varphi$ and $\psi$ are any two members of $\Phi$ which do not both lie in a positive or negative part of a fiber, their product is commutative and is equal to the one in the fiber ahead in this ordering.

The remainder of this section is concerned with Theorem 4.6. Let $L_{\infty}^{R}$ be the real space of equivalence classes of essentially-bounded and real-valued $\mathscr{L}$-measurable functions on $I$ with essential supremum norm $N_{\infty}(\cdot)$. Let $M_{\infty}^{P}\left(M_{\infty}\right)$ denote the space of all real-valued (complex-valued), bounded and $\mathscr{L}$-measurable functions on $I$ with supremum norm.

THEOREM 4.6. There exists a function $U: I \rightarrow \Phi$ such that

(a) $U(t) \in F_{t}^{+}$if $0 \leqq t<1$,

(b) $U(t)(f)=f(t)$ except on a Lebesgue null set depending on $f$, for each $f \in L_{\infty}$,

(c) $\sup \{|U(t)(f)|: t \in[0,1)\}=N_{\infty}(f), f \in L_{\infty}$,

(d) $U(\cdot): L_{\infty} \rightarrow M_{\infty}$ is an algebraic isomorphism,

(e) If $f$ is continuous from the right in $[0,1)$, then $U(t)(f)=f(t)$.

Proof. Let $f \in M_{\infty}^{R}$ and let

$$
\begin{array}{ll}
p(f)(t)=\varlimsup_{n} n \int_{t}^{t+1 / n} f(s) d s, & t \in[0,1), \\
p(f)(1) & =\varlimsup_{n} n \int_{1-1 / n}^{1} f(s) d s .
\end{array}
$$

Then $p(f+g)(t) \leqq p(f)(t)+p(g)(t)$ if $f, g \in M_{\infty}^{R}, t \in I$, and if $\alpha \geqq 0$, $p(\alpha f)(t)=\alpha p(f)(t)$. By the Hahn-Banach theorem, for $t \in I$, there is a linear functional $G(\cdot)(t)$ on $M_{\infty}^{R}$ to the real numbers such that $G(f)(t) \leqq p(f)(t)$. Since $-p(-f)(t) \leqq G(f)(t)$, 


$$
\lim _{n} n \int_{t}^{t+1 / n} f(s) d s \leqq G(f)(t) \leqq \varlimsup_{n} n \int_{t}^{t+1 / n} f(s) d s, \quad t \in[0,1)
$$

and a similar formula holds for $G(f)(1)$. By Lebesgue's differentiation theorem, $G(f)(t)=f(t)$ for almost all $t \in I$ and by completeness of $\mathscr{L}, G$ maps $M_{\infty}^{R}$ into itself. The properties of $G$ include: (i) $G(f)=f$ a.e. on $I$, (ii) $G(1)=1$, (iii) $f=0$ a.e. implies $G(f)=0$, (iv) $f \geqq 0$ implies $G(f) \geqq 0, \quad$ (v) $G$ is linear. If $G$ were also multiplicative the proof could be concluded. A. and C. Ionescu Tulcea have given an elegant proof that a closely related mapping is multiplicative in [8, Prop. 4]. Following their proof, let $\theta^{\prime}, \theta^{\prime \prime}$ be set mappings of $\mathscr{L}$ into $\mathscr{L}$ given by

$$
\theta^{\prime}(E)=\left[t: G\left(k_{E}\right)(t)=1\right], \theta^{\prime \prime}(E)=\left[t: G\left(k_{E}\right)(t) \neq 0\right] .
$$

They show that the convex set consisting of all mappings $G^{\prime}$ of $M_{\infty}^{R}$ into itself which satisfy properties (i) through (v) and which also satisfy

$$
k_{\theta^{\prime}(E)} \leqq G^{\prime}\left(k_{E}\right) \leqq k_{\theta^{\prime \prime}(E)},
$$

$E \in \mathscr{L}$,

has an extreme point $H$ and that $H$ is multiplicative on $M_{\infty}^{R}$. Since $H$ is defined on $L_{\infty}^{R}$ by (iii), the map $f \rightarrow H(f)(t), t \in I$, is a multiplicative linear functional on $L_{\infty}^{R}$. If we set $U(t)(f)=H\left(f_{1}\right)(t)+i H\left(f_{2}\right)(t)$ for $f=f_{1}+i f_{2}$ in the complex space $L_{\infty}$, where $f_{1}, f_{2}$ are real, then $U(t)$ is multiplicative on $L_{\infty}$. Formula (4.6.4) holds for $U$ because it is true for $H$. By (4.6.2) through (4.6.4), $U(t)$ must assume the value 1 on $k_{(t, 1)}$ if $t \in[0,1)$. Hence, $U(t) \in F_{t}^{+}$as asserted. Part (c) is easy to verify. For part (e), suppose $t \in[0,1)$ and that $f$ has a limit from the right at $t$. Then

$$
\begin{aligned}
|U(t)(f)-f(t+0)| & =\left|\int_{I}\{f(s)-f(t+0)\} d \varphi_{t}^{+}(s)\right| \\
& \leqq \int_{t}^{t+\varepsilon}|f(s)-f(t+0)| d \varphi_{t}^{+}(s)
\end{aligned}
$$

for each $\varepsilon>0$. Hence, $U(t)(f)=f(t+0)$.

The following corollary will be needed in the last section.

CoRollaRy 4.7. If $f \in L_{\infty}$ and $U(t+0)(f)$ exists for $t \in[0,1)$, then $U(t+0)(f)=U(t)(f)$.

Proof. Let $g(s)=U(s)(f), s \in[0,1)$. As in the proof of part (e) above, $U(t)(g)=g(t+0)$. Since $f=g$ almost everywhere, $U(s)(f)=$ $U(s)(g)$ for all $s \in[0,1)$. In particular, $g(t)=g(t+0)$.

5. Functions of bounded variation. Let $B V_{0}$ denote the algebra of complex-valued functions of bounded variation defined on $I=[0,1]$, 
which are zero at 0 , with the usual operations for functions. In this section a quotient algebra of $A C_{0}^{* *}$ will be identified with $B V_{0}$.

Let $A$ be a commutative Banach algebra. Let $Y$ be the closed linear manifold in $A^{*}$ which is generated by the multiplicative linear functionals $\mu$ on $A$. Let

$$
Y^{\perp}=\left[\xi \in A^{* *}: \xi(\mu)=0\right] \text {. }
$$

Civin and Yood [3] have shown that (i) $Y^{\perp}$ is a closed two-sided ideal in $A^{* *}$, (ii) $A^{* *} / Y^{\perp}$ is a commutative and semi-simple Banach algebra and (iii) $\hat{\mu}$, the canonical image of $\mu$ in $A^{* * *}$, is a multiplicative linear functional on $A^{* *}$ whenever $\mu$ is multiplicative on $A$.

We recall (Lemma 3.2) that the nonzero multiplicative linear functionals on $A C_{0}$ are given by point evaluations $\mu_{t}(a)=\alpha(t), t \in(0,1]$, $a \in A C_{0}$. Let $Y$, as above, denote the closed subspace of $A C_{0}^{*}$ generated by the $\mu_{t}^{\prime}$ 's. First we identify $Y$. Let $\Sigma$ denote the algebra of all finite unions of intervals of the form $[s, t)$ for $0 \leqq s<t \leqq 1$. Let $B=B\{[0,1), \Sigma\}$ denote the Banach space of all uniform limits of complex linear combinations of characteristic functions of sets from $\Sigma$ with the norm $\|f\|_{B}=\sup \{|f(t)|: t \in[0,1)\}, f \in B$. Let $Y_{\infty}$ denote the closed linear manifold in $L_{\infty}$ which corresponds to $Y$ in $A C_{0}^{*}$ under the isometric isomorphism between $A C_{0}^{*}$ and $L_{\infty}$ mentioned in Section 3 .

Lemma 5.1. The spaces $Y_{\infty}$ and $B$ are isometrically isomorphic under the map $U(\cdot)$ of Theorem 4.6.

Proof. By (3.2.1), if $t \in[0,1)$, then

$$
\mu_{t}(\alpha)=\int_{I} k_{50, t)}(u) a^{\prime}(u) d u, \quad a \in A C_{0} .
$$

Thus, point evaluations in $A C_{0}^{*}$ correspond to equivalence classes in $L_{\infty}$ which contain characteristic functions of the form $k_{[0, t)}$. Hence, $Y_{\infty}$ is the closed linear manifold in $L_{\infty}$ generated by such equivalence classes. Since $k_{(0, t)}$ is continuous from the right, its equivalence class is mapped into $k_{[0, t)} \in M_{\infty}$ under $U(\cdot)$. Hence, $U(\cdot)$ carries $Y_{\infty}$ onto $B$ and is clearly an isometric isomorphism.

Let $\overline{b a}$ denote the complex space $b a\{[0,1), \Sigma\}$ consisting of all finitely additive, complex-valued set functions $\gamma$ defined on $\Sigma$ for which $\operatorname{Var}_{\Sigma}(\gamma,[0,1))$ is finite. It is well known that $\overline{b a}$ is isometrically isomorphic with $B^{*}$ under the correspondence

$$
x^{*}(f)=\int_{[0,1)} f(s) d \gamma(s), \quad x^{*} \in B^{*}, \gamma \in \overline{b a},
$$

where the norm of $x^{*}$ is equal to the total variation of $\gamma$. A nice 
discussion of this integral may be found in $[7 ; 4$, p. 258]. Since $Y$ (which we identify with $Y_{\infty}$ ) and $B$ are isometrically isomorphic and $Y^{*}$ and $A C_{0}^{* *} / Y^{\perp}$ are isometrically isomorphic by general principles, we conclude that $A C_{0}^{* *} / Y^{\perp}$ and $\overline{b a}$ are isometrically isomorphic.

THEOREM 5.2. With the usual point-wise multiplication, $B V_{0}$ is a Banach algebra under the norm $\operatorname{Var}_{I}(\cdot)$ and is isometrically algebraically isomorphic with the quotient algebra $A C_{0}^{* *} / Y^{\perp}$.

Proof. If $g \in B V_{0}$ and we define $\gamma_{g}([s, t))=g(t)-g(s)$ for $0 \leqq$ $s<t \leqq 1$, we obtain a member of $\overline{b a}$. It is obvious that $\operatorname{Var}_{I}(g)=$ $\operatorname{Var}_{\Sigma}\left(\gamma_{g},[0,1)\right)$. Conversely, for $\gamma \in \overline{b a}$ we may define $g_{\gamma}(t)=\gamma([0, t))$ for $0<t \leqq 1, g_{\gamma}(0)=0$, to obtain a member of $B V_{0}$. It follows that $B V_{0}, \overline{b a}$ and the quotient algebra are isometrically isomorphic as Banach spaces. It remains to show that the multiplication induced in $B V_{0}$ from the quotient algebra is the usual pointwise multiplication of functions. It is apparent from the Hahn-Banach theorem and the various isometric isomorphisms mentioned above that set functions in $\overline{b a}$ arise precisely from set functions in $b a$ by restriction to the subalgebra $\Sigma \subset \mathscr{L}$. Thus, a general member of $B V_{0}$ may be viewed as

$$
g_{\xi}(t)=\xi([0, t)), g_{\xi}(0)=0, \quad \xi \in A C_{0}^{* *} .
$$

Let $\eta \in A C_{0}^{* *}$. By remark (iii) in the second paragraph of this section, $(\xi \odot \eta)\left(\mu_{t}\right)=\xi\left(\mu_{t}\right) \eta\left(\mu_{t}\right)$ for point evaluations $\mu_{t}$ on $A C_{0}$. On the other hand,

$$
\xi\left(\mu_{t}\right)=\int_{I} k_{[0, t)}(u) d \xi(u)=\xi([0, t))
$$

and similarly, $\eta\left(\mu_{t}\right)=\eta([0, t))$. Thus, if $g_{\xi \circledast \eta}(t)=(\xi \odot \eta)([0, t))$ corresponds to $\xi \odot \eta$, considered as a member of $\overline{b a}$, then $g_{\xi \odot \eta}(t)=g_{\xi}(t) g_{\eta}(t)$.

The noncommutativity of $A C_{0}^{* *}$ was shown in Theorem 4.5. Another proof of this fact and a proof that $A C_{0}^{* *}$ is not semi-simple has been given by Gulick [6] based upon methods of Civin and Yood.

6. Extension of a homomorphism. Let $A$ be a Banach algebra and suppose $\rho_{0}$ is a bounded homomorphism of $A$ into $B(X)$, the algebra of bounded linear operators on a Banach space $X$. Under the natural embedding $a \rightarrow \hat{a}$ we may consider $A$ as a subalgebra of $A^{* *}$ with Arens multiplication. In this section we consider the problem of extending $\rho_{0}$ to the larger algebra. We recall that a net $\left\{T_{\alpha}\right\}$ converges to $T$ in the weak operator topology in $B(X)$ if and only if $x^{*} T_{\alpha} x \rightarrow$ $x^{*} T x$ for each $x^{*} \in X^{*}$ and each $x \in X$. 
THEOREM 6.1. If $X$ is reflexive, $\rho_{0}$ has an extension to a homomorphism $\rho$ of $A^{* *} B(X)$ such that

(i) $\left\|\rho_{0}\right\|=\|\rho\|$,

(ii) $\rho$ is continuous from $A^{* *}$ with the weak*-topology into $B(X)$ with the weak operator topology.

Moreover, $\rho$ is unique among all extensions of $\rho_{0}$ having property (ii).

Proof. Define the bilinear map $\Delta: X^{*} \times X \rightarrow A^{*}$ by $\left(x^{*} \Delta x\right)(a)=$ $x^{*} \rho_{0}(\alpha) x$ for $a \in A, x^{*} \in X^{*}, x \in X$. Then $\Delta$ is bounded by $\left\|\rho_{0}\right\|$ and the bilinear adjoint $\Delta^{*}$ is given by $\left(\xi \Delta^{*} x^{*}\right)(x)=\xi\left(x^{*} \Delta x\right), \xi \in A^{* *}$ (Section 2 ). For fixed $\xi \in A^{* *}$, this gives a linear map $\rho_{1}(\xi): x^{*} \rightarrow \xi \Delta^{*} x^{*}$ of $X^{*}$ into $X^{*}$ and $\left|\rho_{1}(\xi) x^{*} x\right|=\left|\left(\xi \Delta^{*} x^{*}\right) x\right|=\left|\xi\left(x^{*} \Delta x\right)\right| \leqq\|\xi\|\left\|\rho_{0}\right\|\left\|x^{*}\right\|\|x\|$. Therefore, $\left\|\rho_{1}(\xi)\right\| \leqq\|\xi\|\left\|\rho_{0}\right\|$ and $\rho_{1}(\xi)$ is in $B\left(X^{*}\right)$. Since $X$ is reflexive, the operator adjoint $\rho(\xi)$ of $\rho_{1}(\xi)$ carries $X$ into $X$, is an operator in $B(X)$, and satisfies

$$
x^{*} \rho(\xi) x=\xi(x * \Delta x)
$$

for all $x \in X$ and $x^{*} \in X^{*}$. It is also obvious that $\|\rho(\xi)\| \leqq\|\xi\|\left\|\rho_{0}\right\|$. Thus, $\rho$ clearly satisfies (i) and is a linear extension of $\rho_{0}$ to $A^{* *}$. Because $x^{*} \Delta x \in A^{*}$, it is obvious from Formula (6.1.1) that $\rho$ satisfies (ii). It remains to show that $\rho$ is multiplicative and is unique subject to condition (ii). Since each $\xi \in A^{* *}$ is the weak*-limit of a net $\left\{\hat{a}_{\alpha}\right\}$ from $\hat{A}$, an obvious computation establishes that Formula (6.1.1) may be expressed as

$$
x^{*} \rho(\xi) x=\lim _{\alpha} x^{*} \rho_{0}\left(a_{\alpha}\right) x, \quad \xi=\text { weak }^{*}-\lim _{\alpha} \hat{a}_{\alpha},
$$

for all $x \in X$ and $x^{*} \in X^{*}$. If $\rho^{\prime}$ is any mapping of $A^{* *}$ into $B(X)$ which extends $\rho_{0}$ and which satisfies (ii), it is easily seen, with the aid of Formula (6.1.2) that $\rho=\rho^{\prime}$. Now let $\xi=$ weak $^{*}-\lim _{\alpha} \hat{a}_{\alpha}$ and let $\eta=$ weak $^{*}-\lim _{\beta} b_{\beta}$ where $a_{\alpha}$ and $b_{\beta}$ are in $A$. Then $\hat{a}_{\alpha} \odot \eta=$ weak ${ }^{*}-\lim _{\beta} \hat{a}_{\alpha} \odot \hat{b}_{\beta}$ (see Section 2). By Formula (6.1.2), $x^{*} \rho\left(\hat{a}_{\alpha} \odot \eta\right) x=$ $\lim _{\beta} x^{*} \rho\left(\hat{a}_{\alpha} \odot \hat{b}_{\beta}\right) x=\lim _{\beta} x^{*} \rho_{0}\left(a_{\alpha} b_{\beta}\right) x=\lim _{\beta} x^{*} \rho_{0}\left(a_{\alpha}\right) \rho_{0}\left(b_{\beta}\right) x=x^{*} \rho_{0}\left(a_{\alpha}\right) \rho(\eta) x$. Since $\xi \odot \eta=$ weak $^{*}-\lim _{\alpha} \hat{a}_{\alpha} \odot \eta$, using Formula (6.1.2) again, we get $x^{*} \rho(\xi \odot \eta) x=\lim _{\alpha} x^{*} \rho_{0}\left(a_{\alpha}\right) \rho(\eta) x=x^{*} \rho(\xi) \rho(\eta) x$. Since this holds for each $x$ and $x^{*}, \rho(\xi \odot \eta)=\rho(\xi) \rho(\eta)$.

With minor modifications the proof of Theorem 6.1 establishes the following variant on that theorem

THEOREM 6.2. If $A$ is a commutative Banach algebra and $\rho_{0}$ is a bounded homomorphism of $A$ into $B\left(Y^{*}\right)$ for some Banach space $Y$, then $\rho_{0}$ has an extension to a homomorphism $\rho$ of $A^{* *}$ into $B\left(Y^{*}\right)$ such that

(i) $\left\|\rho_{0}\right\|=\|\rho\|$, 
(ii) If $\xi=$ weak $^{*}-\lim _{\alpha} \hat{\alpha}_{\alpha}, \xi \in A^{* *}, a_{\alpha} \in A$, then $\rho(\xi) y^{*} y=$ $\lim _{\alpha} \rho_{0}\left(a_{\alpha}\right) y^{*} y$ for all $y \in Y$ and $y^{*} \in Y^{*}$.

Moreover, $\rho$ is unique among all extensions of $\rho_{0}$ having property (ii).

We shall only be interested in the reflexive situation as in Theorem 6.1. Let $M$ denote the closed linear manifold in $A^{*}$ generated by linear functionals of the form $x^{*} \Delta x, x^{*} \in X^{*}, x \in X$. It is evident from Formula (6.1.1) that the kernel of $\rho$ is $M^{\perp}$. Formula (6.1.2) implies that the range of the extension $\rho$ is contained in the closure of the range of $\rho_{0}$ in the weak operator topology. Thus, if $A$ is commutative, the range of $\rho$ is a commutative algebra of operators in $B(X)$.

The question arises whether Theorem 6.1 can be applied to $\rho$ in order to obtain a further extension to $A^{* * * *}$. There are no further nontrivial extensions by the method of Theorem 6.1.

7. Well-bounded operators. Let $X$ be a reflexive Banach space. Let $T$ be the well-bounded operator mentioned in Section 1 with an operational calculus $a \rightarrow a(T)$ where $a \in A C(I)$ and

$$
\|a(T)\| \leqq K\left\{|a(0)|+\operatorname{Var}_{I}(a)\right\}, \quad I=[0,1] .
$$

This operational calculus is uniquely determined by its values on complex polynomials as these are dense in $A C(I)$ with the norm (1.0.1) (cf. [14, Lemma 2.1]).

Let $\rho_{0}$ be the homomorphism $\rho_{0}(\alpha)=a(T)$ induced on $A C_{0}$ by the operational calculus. As in Theorem 6.1, $\rho_{0}$ has a unique extension to a bounded homomorphism $\rho$ of $A C_{0}^{* *}$ into $B(X)$ such that $x^{*} \rho(\xi) x=$ $\xi\left(x^{*} \Delta x\right)$ for $x \in X, x^{*} \in X^{*}$ and $\xi \in A C_{0}^{*}$. The existence of $\rho$ and the $\operatorname{map} t \rightarrow U(t)$ of Theorem 4.6 lead to the spectral theorem for $T$. The space $Y$ and $B$ mentioned below are defined in Section 5 .

Theorem 7.1. (i) If $x \in X, x^{*} \in X^{*}$, then $x^{*} \Delta x \in Y$.

(ii) For each $x \in X$, the vector valued function $t \rightarrow \rho(U(t)) x$ is continuous from the right in $[0,1)$.

Proof. By Theorem 4.4, if $0 \leqq s \leqq t<1, U(s) \odot U(t)=U(t) \odot U(s)=$ $U(t)$. Hence, $\{\rho(U(t))\}$ for $t \in[0,1)$ is a bounded and nonincreasing family of projections in $B(X)$. Since $X$ is reflexive a theorem of Lorch [11, Theorem 3.2] states that the function $t \rightarrow \rho(U(t)) x$ has a limit from the right at each point of $[0,1)$ and has a limit from the left at each point of $(0,1]$. If $x^{*} \in X^{*}$, the function $t \rightarrow x^{*} \rho(U(t)) x=$ $U(t)\left(x^{*} \Delta x\right)$ has the same limit properties. By Corollary 4.7 this function is continuous from the right in $[0,1)$. The space $B$ can be characterized as the space of all bounded complex-valued functions defined and 
continuous from the right in $[0,1)$ and which have limits from the left at points of $(0,1]$ (see [7], Theorem 4.5]). Therefore, $U(\cdot)\left(x^{*} \Delta x\right)$ is in $B$. By Lemma $5.1, x^{*} \Delta x$ is in $Y$ (we identify $Y_{\infty}$ with $Y$ ). This proves (i). To prove (ii), let $s$ be fixed in $[0,1)$. By Lorch's theorem, there is a $z \in X$ such that $z=\lim _{t \rightarrow s+0} \rho(U(t)) x$. Since $x^{*} z=$ $\lim _{t \rightarrow s+0} x^{*} \rho(U(t)) x=x^{*} \rho(U(s)) x$ for each $x^{*} \in X^{*}$, then $z=(U(s)) x$.

COROLLARY 7.2. The kernel of $\rho$ contains $Y^{\perp}$.

Proof. Since $M$, the closed linear manifold in $A C_{0}^{*}$ generated by the functionals $x^{*} \Delta x$ is contained in $Y$, the kernel of $\rho, M^{\perp}$, contains $Y^{\perp}$.

Definition 7.3. For each $t \in[0,1)$ choose a member $V(t)$ from the positive side of the fiber $F_{t}^{+}$(Sec. 4) and let

$$
E_{t}=\left\{\begin{array}{l}
0 \text { if }-\infty<t<0 \\
I-\rho(V(t)) \text { if } 0 \leqq t<1 \\
I \text { if } 1 \leqq t<\infty
\end{array}\right.
$$

where 0 and $I$ are the zero and identity operators in $B(X)$.

THEOREM 7.4. The family of projections $\left\{E_{t}\right\}$ does not depend upon the choice $\{V(t)\}$ (when $X$ is reflexive) and satisfies

(i) $\left\|E_{t}\right\| \leqq K+1$,

(ii) $E_{s} E_{t}=E_{m i n(s, t)}$,

(iii) $\lim _{t \rightarrow s+0} E_{t} x=E_{s} x$ for each $x \in X$,

(iv) $E_{t}=0$ if $t<0, E_{t}=I$ if $t \geqq 1$.

Proof. Let $t \in[0,1)$ be fixed. By the definition of $F_{t}^{+}$any two members, say $V(t)$ and $U(t)$ agree as set functions on intervals of the form $[0, s)$ for $0<s \leqq 1$. Hence, as functionals on $A C_{0}^{*}, V(t)\left(\mu_{s}\right)=$ $U(t)\left(\mu_{s}\right)$ for each point evaluation $\mu_{s}$ on $A C_{0}$ (see Formula (5.2.1). By linearity and continuity, $V(t)$ and $U(t)$ agree on $Y$. Since $X$ is reflexive, $\rho(V(t))=\rho(U(t))$ by Corollary 7.2. The second statement is clear by Definition 7.3 and Theorem 4.4. The third was shown in Theorem 7.1 (ii).

At this stage it is an easy matter to obtain spectral integrals for a $T$ satisfying (7.0.1). Let $a=(a-a(0) e)+a(0) e$ be in $A C(I)=$ $A C_{0} \oplus\{\lambda e\}$. Let $x^{*} \in X^{*}$ and $x \in X$. Then

$$
\begin{aligned}
x^{*} a(T) x & =x^{*} x a(0)+x^{*} \rho_{0}(a-a(0) e) x \\
& =x^{*} x a(0)+\left(x^{*} \Delta x\right)(a-a(0) e) \\
& =x^{*} x a(0)+\int_{I}\left(x^{*} \Delta x\right)(s) a^{\prime}(s) d s
\end{aligned}
$$


by Formula (3.2.1). By Theorem 4.6 we may replace $\left(x^{*} \Delta x\right)(s)$ by $U(s)\left(x^{*} \Delta x\right)$ and by Definition 7.3 we have

$$
x^{*} a(T) x=x^{*} x a(0)+\int_{I} x^{*}\left(I-E_{s}\right) x a^{\prime}(s) d s .
$$

This Lebesgue integral is a Riemann-Stieltjes integral by the absolute continuity of $a$ and because the integrand, a member of $B$, has at most a countable set of discontinuities. Also, by a slight generalization of a theorem of Graves ([5, Theorem 1]) it can be shown that the Riemann-Stieltjes integral

$$
\int_{J}\left(I-E_{s}\right) x d a(s)
$$

exists in $X$ whenever $a$ is continuous and of bounded variation on the closed and bounded interval $J$. ( $A$ variant of the proof given in [5] is valid for such an $a$ because the vector valued function $t \rightarrow E_{t} x$ has at most a countable set of discontinuities by right continuity $([14, \mathrm{p}$. $330])$ ). We may suppose that $a$ is absolutely continuous on $[-\varepsilon, 1]$, $\varepsilon>0$. By these remarks, we may remove the $x^{*}$ from (7.4.1), integrate by parts on $[-\varepsilon, 1]$ and let $\varepsilon \rightarrow 0$ to obtain the strong integral

$$
a(T) x=\int_{0 \_}^{1} a(s) d E_{s} x, \quad a \in A C(I), x \in X .
$$

If the unit function $e$ is adjoined to $B V_{0}$ we obtain $B V(I)=$ $B V_{0} \oplus\{\lambda e\}$, the Banach algebra of complex-valued functions of bounded variation on $I$ with the norm $|g(0)|+\operatorname{Var}_{I}(g), g \in B V(I)$. As in the proof of Theorem 5.2, members of $B V_{0}$ arise from set functions $\xi \in A C_{0}^{* *}$ by the correspondence $g_{\xi}(t)=\xi([0, t))$ for $0<t \leqq 1, g_{\xi}(0)=0$. The notion of integration of functions in $B$ with respect to set functions in $\overline{b a}$ as in (5.1.1) is essentially the same as that given in the paragraph after Formula (3.2.1). The only difference is that $\Sigma$ replaces $\mathscr{L}$ and the sup norm on $[0,1)$ replaces $N_{\infty}(\cdot)$. With this in mind it is easy to see that

$$
\int_{[0,1)} f(t) d g_{\xi}(t)=\int_{I} f(t) d \xi(t)
$$

whenever $f \in B$. Thus, proceeding as in the derivation of (7.4.1), one obtains the operational calculus $g_{\xi} \rightarrow g_{\xi}(T)$ of $B V_{0}$ into $B(X)$ in the weak form

$$
x^{*} \rho(\xi) x=\int_{[0,1)} x^{*}\left(I-E_{t}\right) x d g_{\xi}(t)=x^{*} g_{\xi}(T) x .
$$

This calculus may be extended to $B V_{0} \oplus\{\lambda e\}$ in the obvious way. 
In conclusion, the role of the negative sides of the fibers will be clarified. Let $t \in(0,1]$ be fixed. For each $s \in[0, t)$ choose a member $\varphi_{s}^{+} \in F_{s}^{+}$. Let $\varphi_{t}^{-} \in F_{t}^{-}$be choosen. Let $0<r \leqq 1$. It is easy to verify that $\lim _{s \rightarrow t-0} \varphi_{s}^{+}\left(\mu_{r}\right)=\lim _{s \rightarrow t-0} \varphi_{s}^{+}([0, r))=\varphi_{t}^{-}([0, r))=\varphi_{t}^{-}\left(\mu_{r}\right)$ for point evaluations $\mu_{r}$. The limit also holds on finite linear combinations of the $\mu_{r}$ and, hence, it holds on $Y$. Therefore, $x^{*} \rho\left(\varphi_{t}^{-}\right) x=\varphi_{t}^{-}\left(x^{*} \Delta x\right)=$ $\lim _{s \rightarrow t-0} \varphi_{s}^{+}\left(x^{*} \Delta x\right)=\lim _{s \rightarrow t-0} x^{*}\left(I-E_{s}\right) x=x^{*}\left(I-E_{t \rightarrow 0}\right) x$ for each $x \in X$ and $x^{*} \in X^{*}$. Hence, $\rho\left(\varphi_{t}^{-}\right)=I-E_{t-0}$.

\section{BIBLIOGRAPHY}

1. R. Arens, Operations induced in function classes, Monat. für Math. 55 (1951), 1-19.

2. R. Arens, The adjoint of a bilinear operation, Proc. Amer. Math. Soc. 2 (1951), 839-848.

3. P. Civin and B. Yood, The second conjugate space of a Banach algebra as an algebra, Pacific J. Math. 11 (1961), 847-870.

4. N. Dunford and J. Schwartz, Linear operators, Part I, Interscience Publishers, Inc., New York, 1958.

5. L. M. Graves, Riemann integration and Taylor's theorem in general analysis, Trans. Amer. Math. Soc. 29 (1927), 163-177.

6. S. L. Gulick, 3d, The bidual of a locally multiplicatively-convex algebra, Thesis, Yale University, 1963.

7. E. Hewitt, A problem concerning finitely additive measures Mat. Tidsskr. B 1951 (1951), 81-94.

8. A. Ionescu Tulcea and C. Ionescu Tulcea, On the lifting property (I), J. of Math. Analysis and Applications 3 (1961), 537-546.

9. H. Kamowitz, Cohomology groups of commutative Banach algebras, Trans. Amer. Math. Soc. 102 (1962), 352-372.

10. L. H. Loomis, An introduction to harmonic analysis, D. Van Nostrand Co., New York, 1953.

11. E. R. Lorch, On a calculus of operators in reflexive vector spaces, Trans. Amer. Math. Soc. 45 (1939), 217-234.

12. J. R. Ringrose, On well-bounded operators, J. Australian Math. Soc. 1 (1960), $334-343$.

13. - On well-bounded operators, II, Proc. London Math. Soc. (3) 13 (1963), 613-638.

14. D. R. Smart, Conditionally convergent spectral expansions, J. Australian Math. Soc. 1 (1960), 319-333.

15. G. L. Krabbe, Stieltjes integration, spectral analysis, and the locally-convex algebra $(B V)$, Bull. Amer. Math. Soc. 71 (1965), 184-189.

UNIVERSITY OF OREGON

AND

San Jose State College 


\section{PACIFIC JOURNAL OF MATHEMATICS}

\section{EDITORS}

\author{
H. SAMELSON \\ Stanford University \\ Stanford, California \\ R. M. BLUMENTHAL \\ University of Washington \\ Seattle, Washington 98105
}

\author{
*J. DUGUNDJI \\ University of Southern California \\ Los Angeles, California 90007
}

RICHARD ARENS

University of California Los Angeles, California 90024

\section{ASSOCIATE EDITORS}
E. F. BECKENBACH
B. H. NEUMANN
F. WOLF
K. YoSIDA

\section{SUPPORTING INSTITUTIONS}

\author{
UNIVERSITY OF BRITISH COLUMBIA \\ CALIFORNIA INSTITUTE OF TECHNOLOGY \\ UNIVERSITY OF CALIFORNIA \\ MONTANA STATE UNIVERSITY \\ UNIVERSITY OF NEVADA \\ NEW MEXICO STATE UNIVERSITY \\ OREGON STATE UNIVERSITY \\ UNIVERSITY OF OREGON \\ OSAKA UNIVERSITY \\ UNIVERSITY OF SOUTHERN CALIFORNIA
}

\author{
STANFORD UNIVERSITY \\ UNIVERSITY OF TOKYO \\ UNIVERSITY OF UTAH \\ WASHINGTON STATE UNIVERSITY \\ UNIVERSITY OF WASHINGTON \\ * * * * \\ AMERICAN MATHEMATICAL SOCIETY \\ CHEVRON RESEARCH CORPORATION \\ TRW SYSTEMS \\ NAVAL ORDNANCE TEST STATION
}

Mathematical papers intended for publication in the Pacific Journal of Mathematics should be typewritten (double spaced). The first paragraph or two must be capable of being used separately as a synopsis of the entire paper. It should not contain references to the bibliography. Manuscripts may be sent to any one of the four editors. All other communications to the editors should be addressed to the managing editor, Richard Arens at the University of California, Los Angeles, California 90024.

50 reprints per author of each article are furnished free of charge; additional copies may be obtained at cost in multiples of 50 .

The Pacific Journal of Mathematics is published monthly. Effective with Volume 16 the price per volume (3 numbers) is $\$ 8.00$; single issues, $\$ 3.00$. Special price for current issues to individual faculty members of supporting institutions and to individual members of the American Mathematical Society: $\$ 4.00$ per volume; single issues $\$ 1.50$. Back numbers are available.

Subscriptions, orders for back numbers, and changes of address should be sent to Pacific Journal of Mathematics, 103 Highland Boulevard, Berkeley 8, California.

Printed at Kokusai Bunken Insatsusha (International Academic Printing Co., Ltd.), No. 6, 2-chome, Fujimi-cho, Chiyoda-ku, Tokyo, Japan.

\section{PUBLISHED BY PACIFIC JOURNAL OF MATHEMATICS, A NON-PROFIT CORPORATION}

The Supporting Institutions listed above contribute to the cost of publication of this Journal, but they are not owners or publishers and have no responsibility for its content or policies.

* Paul A. White, Acting Editor until J. Dugundji returns. 


\section{Pacific Journal of Mathematics}

\section{Vol. 17, No. 2 \\ February, 1966}

Henry A. Antosiewicz, Boundary value problems for nonlinear ordinary

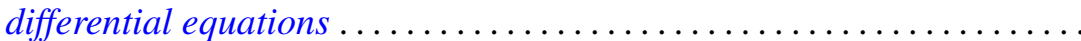

Bernard Werner Levinger and Richard Steven Varga, Minimal Gerschgorin sets. II ........................................

Paul Camion and Alan Jerome Hoffman, On the nonsingularity of complex matrices........................................ 211

J. Chidambaraswamy, Divisibility properties of certain factorials ........ 215

J. Chidambaraswamy, A problem complementary to a problem of Erdös .... 227

John Dauns, Chains of modules with completely reducible quotients ...... 235

Wallace E. Johnson, Existence of half-trajectories in prescribed regions and asymptotic orbital stability ............................ 243

Victor Klee, Paths on polyhedra. II . ........................ 249

Edwin Haena Mookini, Sufficient conditions for an optimal control problem in the calculus of variations ............................ 263

Zane Clinton Motteler, Existence theorems for certain quasi-linear elliptic equations........................................... 279

David Lewis Outcalt, Simple n-associative rings ............... 301

David Joseph Rodabaugh, Some new results on simple algebras ......... 311

Oscar S. Rothaus, Asymptotic properties of groups generation ........... 319

Ernest Edward Shult, Nilpotence of the commutator subgroup in groups admitting fixed point free operator groups .............

William Hall Sills, On absolutely continuous functions and the

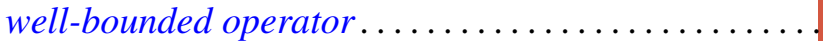

Joseph Gail Stampfli, Which weighted shifts are subnormal ..

Donald Reginald Traylor, Metrizability and completeness in normal Moore spaces 